\title{
ANALYSIS OF CHROMOSOMAL REGIONS CONTROLLING DROUGHT TOLERANCE IN BARELY (HORDEUM VULGARE L.) SEEDLINGS
}

\author{
MOHAMmAdi, A. ${ }^{1}-$ SofAlian, O. ${ }^{*}-$ JAFARY, H. ${ }^{2}-$ AsGHARI, A. ${ }^{1}-$ SHEKARI, F. ${ }^{3}$ \\ ${ }^{I}$ Department of Agronomy and Plant Breeding, Faculty of Agriculture and Natural Resources, \\ University of Mohaghegh Ardabili, Ardabil, Iran \\ (e-mail: mohamadi.arash@gmail.com; phone: +98-912-841-8261) \\ ${ }^{2}$ Plant Protection Research Department, Zanjan Agricultural and Natural Resources Research \\ and Education Center, AREEO, Zanjan, Iran \\ (e-mail: hjafary@gmail.com; phone: +98-912-442-5017) \\ ${ }^{3}$ Department of Agronomy and Plant Breeding, Faculty of Agriculture Sciences, University of \\ Zanjan, Zanjan, Iran \\ (e-mail: faridshekari@yahoo.com; phone: +98-912-242-4829) \\ *Corresponding author \\ e-mail: sofalian@gmail.com; phone: +98-914-403-9186 \\ (Received $17^{\text {th }}$ Sep 2017; accepted $24^{\text {th }}$ May 2018)
}

\begin{abstract}
To locate the loci controlling physiological traits of barley in seedling stage, an experiment was conducted under drought stress and normal irrigation in greenhouse as a completely randomized design with two replications in Iran in 2016. For this purpose, the populations of Cebad Capa (C. Capa) $\times$ Susptrit (S) and Vada (V) $\times$ Susptrit (S) with their parents were separately cultivated under drought stress condition and normal irrigation. 112 and 89 Recombinant Inbred Lines respectively resulted from $\mathrm{V} \times \mathrm{S}$ and C. Capa $\times \mathrm{S}$ were used. Susptrit was used as drought tolerant cultivar and Vada and Cebada Capa were selected as sensitive cultivars. In this experiment Chlorophyll content (CC), relative water content (RWC), shoot dry weight (SDW) and leaf temperature (LT) were measured. For each population and environment, QTL (Quantitative Trait Loci) analysis was done based on composite interval mapping on the lines separately. The results showed the effect of genotype was significant for all traits. 43 locations covering QTLs were identified in both populations and both environments. 15 QTLs were located for C. Capa $\times$ S under drought stress. The identified QTLs under drought stress were identified as three QTLs for RWC on chromosomes 4 and 5, six QTLs for chlorophyll content as two QTLs for CC-30\%FC and two QTLs for CC-50\%FC on chromosomes 3 and 7, two QTLs for CC-20\% FC on chromosomes 4 and 7, three QTLs for SDW on chromosomes 1 and 6, and three QTLs for LT on chromosomes 4, 6, and 7. In contrast, two QTLs on chromosomes 3 and 6 were detected under normal irrigation for SDW. In addition, 13 QTLs were located for $\mathrm{V} \times \mathrm{S}$ under drought stress. 10 QTLs were observed for chlorophyll content as three QTLs for CC-50\%FC on chromosomes 3 and 7, three QTLs for CC-30\%FC on chromosomes 1, 2, and 5, four QTLs for CC-20\%FC on chromosomes 2, 3 and 6. For RWC, there were identified two QTLs on chromosomes 4 and 6, while a QTL was recorded for SDW on chromosomes 6. Our study showed stable QTLs within and between populations, which it can be used in selection process by markers.
\end{abstract}

Keywords: barley, drought stress, mapping, physiological traits, quantitative trait loci

\section{Introduction}

Barley (Hordeum vulgare L.), is a major cereal grain grown in temperate climates globally and the fourth most widely grown small-grain cereal in the world (Ashida et al., 2007). It was one of the first cultivated grains, particularly in Eurasia as early as 13,000 years ago. Barley has been used as animal fodder, as a source of fermentable material for beer and certain distilled beverages, and as a component of various healthy 
foods. It is used in soups and stews, and in barley bread of various cultures. It has high flexibility to various environmental conditions especially for water deficient condition (Ceccarelli, 1987). Assessing the grain yield in areas with intensive stress is the most common method to describe the adaptation of plants under stress conditions (Blum, 1988). Different traits of plants are corresponded to drought stress, but the traits indicating the relative water capacity (RWC) can reveal useful findings for water stress shortage. RWC has been reported as a useful parameter in drought stress by PeltonenSaino and Mäkelä (1995) and Teulat et al. (2003). Gutierrez-Rodriguez et al. (2004) found that there was a significant relationship between chlorophyll content, photosynthesis rate and yield of wheat genotypes cultivated in normal irrigation and water deficit treatments, so the chlorophyll content could be a representative of photosynthesis capacity. Although the analization of some traits could be time consuming, using DNA markers were efficient method for identify QTLs, and selection programmed by marker assisted selection (MAS). Especially in the case of drought stress by identifying certain loci for tolerating sensitive genotypes based on MAS procedure. Molecular methods make a way to produce the drought tolerant cultivars with high capacity of water uptake (Hammam Mohammed, 2004). Many gene loci controlling traits are associated with drought tolerance and they have been evaluated by relative performance of strains (Mackill, 2003). Chen et al. (2004) showed that in barley, eibil gene was caused strong sensitivity to drought condition. Hammam Mohammed (2004) used a new approach based on advanced backcross in barley to identify QTLs for osmotic adjustment, relative water content, carbon isotope discrimination, dry matter yield and seed yield in dry conditions in the greenhouse. Molecular markers were useful and efficient to identifying the controlling factors associated with drought stress and introducing the improved cultivars. Seedling stage as a critical phase in plant establishment is very important in plant growth. Moreover, assessment of traits in early stages which are linked to the adult stage can facilitate early selection of desirable genotypes. Direct selection might not have adequate proficiency for improving yield under drought conditions due to low heritability, polygenic control, presence of epistasis, QTL $\times$ environment interactions $(\mathrm{QTL} \times \mathrm{E})$, and complex mechanisms of drought tolerance. So, in drought tolerance improvement programs, finding molecular markers associated to chromosomal regions which control drought tolerance traits could accelerate breeding programs and raise the selection efficiency (Cattivelli et al., 2002). Determining the number of QTLs controlling quantitative traits is a crucial attempt in plant molecular breeding (Cooper et al., 2009). QTL analysis makes association between continuous variation of the phenotype and genotypic variation of any concerned loci (Collard et al., 2005). Identifying QTLs make possibilities to accomplish MAS programs (Emberini et al., 2009). Climate changes and global warming is a main challenge in present-day agriculture, which are considered as a risk factor for crop plants. Hence, further researches are necessary to obtain the appropriate varieties using molecular breeding. The present study was conducted to determining QTLs associated with some physiological traits involved in drought stress as well as estimating the effects of the QTLs in barley.

\section{Material and methods}

A total of 112 recombinant inbred lines (RILs) of Vada $\times$ Susptrit and 89 RILs of Cebad Capa $\times$ Susptrit were selected. The Susptrit was drought tolerant cultivar, 
whereas Cebada Capa and Vada were sensitive cultivars (Nguyen, 2012). Population of $\mathrm{V} \times \mathrm{S}$ derived from Vada $\times$ Susptrit included 112 lines, where its genetic map with 450 markers consisted of 420 amplified fragment length polymorphism (AFLP) and 24 simple sequence repeats (SSR) markers (Jafary et al., 2006) and also population of C. Capa $\times \mathrm{S}$ from Cebad Capa $\times$ Susptrit consisted of 89 lines with 459 markers included 481 AFLP and 14 SSR markers (Jafary et al., 2008) (Table 1).

Table 1. Characteristics of two barley populations used in this study. "Gene" is related to two morphologic markers include naked vs cowered seed, and two row vs six row ear, also "other markers" indicate restricted fragment length polymorphysm (RFLP) markers

\begin{tabular}{c|c|c|c|c|c|c|c}
\hline \multicolumn{3}{c|}{ Populations } & \multicolumn{5}{c}{ Markers } \\
\hline Population & Type & Number of lines & AFLP & SSR & Gene & $\begin{array}{c}\text { Other } \\
\text { markers }\end{array}$ & Total \\
\hline $\mathrm{V} \times \mathrm{S}$ & RIL & 112 & 420 & 24 & 2 & 4 & 450 \\
$\mathrm{CC} \times \mathrm{S}$ & RIL & 89 & 481 & 14 & 0 & 0 & 459 \\
\hline
\end{tabular}

This study was performed under a completely randomized design in a greenhouse, controled by average temperature $25^{\circ} \mathrm{C}$ and moisture $70 \%$ in Iran in 2016 . The lines of both populations were separately cultivated in $15 \times 25-\mathrm{cm}$ plastic pots. Because of uniformity of the greenhouse environment and soil bedding, each experiment was conducted under a completely randomized design with two replications was. In both populations and parent cultivars, 5 sound seeds were selected and after disinfected with $70 \%$ Ethilic Alcohol, they were cultivated in plastic pots filled with field soil and sand as $1: 1 \mathrm{v} / \mathrm{v}$. The thinning led to reduce of three seedlings per pot. All pots were irrigated uniformly until 4-leaf stage. Standard Hoagland nutrient solution (Table 2) was used in two stages (2-leaf and 4-leaf stages) $200 \mathrm{ml}$ per pot in both stages. The drought stress was imposed at the 4-leaf stage by stopping the irrigation for stress experiments, while irrigated experiments was maintained well-watered. Drought stress was applied up to $20 \%$ of field capacity (FC). In this stage all traits were measured in both experiments.

Table 2. Chemical compounds and those concentrations consist of standard Hoagland nutrient solution, used in this study

\begin{tabular}{c|c|c}
\hline Chemical compound & Chemical formula & Concentration $\left.\mathbf{~ g ~}^{\mathbf{1}}\right)$ \\
\hline Calcium nitrate & $\mathrm{Ca}\left(\mathrm{NO}_{3}\right)_{2} \cdot 4 \mathrm{H}_{2} \mathrm{O}$ & 3 \\
Potassium nitrate & $\mathrm{K}\left(\mathrm{NO}_{3}\right)_{2}$ & 1.25 \\
Magnesium sulphate & $\mathrm{MgSO}_{4}$ & 1.1 \\
Ammonium mono phosphate & $(\mathrm{NH})_{3} \mathrm{PO}_{4}$ & 0.3 \\
\hline
\end{tabular}

\section{Measured traits}

Chlorophyll content $(C C)$ : Chlorophyll content of barley leaves was tested in drought stress during the stress period in 3 stages at $50 \%$ FC, 30\% FC and 20\% FC using SPAD (MINOLTA). In normal experiments, one step was measured. To measure the chlorophyll content of the leaves, the last fully developed leaves and the middle part of the leaves were used. This trait was measured in each of the 3 plants per pot, and average amount was used for further analysis. 
Relative water content $(R W C)$ : Several pieces of the last developing barley leaves were used based on Barr and Weatherley (1962). Sampling was done after 11 AM. Fresh weight of leaf samples was measured immediately. All samples were transported to the laboratory in a tank containing ice bricks. Then, the leaf samples immediately immersed in separate containers containing a sufficient amount of distilled water and were incubated at $25^{\circ} \mathrm{C}$. Turgid weight (TW) was measured after $48 \mathrm{~h}$. Then, the leaf samples envelopes for $24 \mathrm{~h}$ in an oven at $75{ }^{\circ} \mathrm{C}$, the dry weight (DW) of leaf samples were immediately recorded. Finally, RWC was calculated according to Equation 1:

$$
R W C \%=\left[\frac{F W-D W}{T W-D W}\right] \times 100
$$

Shoot dry weight $(S D W)$ : The shoots of three plants in per pot were harvested and dried at $75{ }^{\circ} \mathrm{C}$ for $24 \mathrm{~h}$ and then were weighted and average of three plants were recorded.

Leaf temperature $(L T)$ : LT was measured in populations of C. Capa $\times \mathrm{S}$. The infrared thermometer was used and measurements were taken on the last well developed leaves on every 3 plants in each pot and their averages were recorded.

\section{Statistical analysis}

Analysis of variance and calculation of descriptive statistics were performed using software SPSS 22.

\section{QTL analysis}

QTL analysis software was used for MapQTL 5. This analysis was performed using the automatic cofactor selection (ACS) and the method of multiple-QTL mapping (MQM). To determine LOD threshold, permutation test was used. In order to map the chromosomal software MapChart 2.2 was applied.

\section{Results}

\section{Analysis of variance (ANOVA)}

Analysis of variance showed that there was significant difference $(P \leq 0.01)$ between different genotypes (Tables 3 and 4).

Table 3. Results of combined ANOVA of the traits in population $V \times S$

\begin{tabular}{c|c|c|c|c}
\hline \multicolumn{5}{c}{ MS } \\
\hline Source of variations & df & $\begin{array}{c}\text { Leaf relative } \\
\text { water content }\end{array}$ & Chlorophyll content & Shoot dry weight \\
\hline Environment & 1 & $22652.96^{* *}$ & $431.84^{* *}$ & $1.34^{* *}$ \\
Rep/Environment & 2 & 60.53 & 38.56 & 0.046 \\
Genotype & 111 & $169.79^{* *}$ & $44.68^{* *}$ & $0.011^{* *}$ \\
Genotype $\times$ Environment & 111 & $119.63^{* *}$ & $21.46^{* *}$ & $0.005^{* *}$ \\
Error & 222 & 17.92 & 6.52 & 0.004 \\
\hline
\end{tabular}

Ns, *, and ** indicate no significant, significant at $5 \%$ level, and significant at $1 \%$ level, respectively. MS: mean of squares, df: degree of freedom, Rep: replication 
Table 4. Results of combined ANOVA of the traits in population C. Capa $\times S$

\begin{tabular}{c|c|c|c|c|c}
\hline \multicolumn{6}{|c}{ MS } \\
\hline Source of variations & df & $\begin{array}{c}\text { Leaf relative water } \\
\text { content }\end{array}$ & $\begin{array}{c}\text { Chlorophyll } \\
\text { content }\end{array}$ & $\begin{array}{c}\text { Leaf } \\
\text { temperature }\end{array}$ & $\begin{array}{c}\text { Shoot dry } \\
\text { weight }\end{array}$ \\
\hline Environment & 1 & $26610.14^{* *}$ & $95.01^{* *}$ & $310.17^{* *}$ & $1.103^{* *}$ \\
Rep/Environment & 2 & 208.91 & 16.98 & 11.59 & 0.019 \\
Genotype & 88 & $89.41^{* *}$ & $45.41^{* *}$ & $3.89^{* *}$ & $0.013^{* *}$ \\
Genotype $\times$ Environment & 88 & $75.66^{* *}$ & $17.53^{* *}$ & $3.30^{* *}$ & $0.006^{* *}$ \\
Error & 176 & 31.95 & 4.32 & 1.50 & 0.003 \\
\hline
\end{tabular}

Ns, *, and ** indicate no significant, significant at $5 \%$ level, and significant at $1 \%$ level, respectively. MS: mean of squares, df: degree of freedom, Rep: replication

The effects of the environment and genotype $\times$ environment on all traits in both crosses were significant. In addition, a significant genetic diversity between the traits was observed. Discriptive statistics of traits in parents and RILs of two populations in drought stress and irrigation experiments, were showed in Tables 5 and 6 , respectively.

Table 5. Descriptive statistics for traits in populations of C. Capa $\times S$ and $V \times S$ under drought stress experiments

\begin{tabular}{|c|c|c|c|c|c|c|c|}
\hline & \multirow{2}{*}{ Traits } & \multicolumn{2}{|c|}{ Means of parents } & \multicolumn{4}{|c|}{ Means of population } \\
\hline & & $\mathbf{S}$ & C. Capa & Mean & SD & Minimum & Maximum \\
\hline \multirow{8}{*}{$\begin{array}{l}\text { Drought stress } \\
\text { condition } \\
(\text { C. Capa } \times \text { S })\end{array}$} & RWC & 90.4 & 63.88 & 70.30 & 8.55 & 51.76 & 86.68 \\
\hline & $\mathrm{CC} 50 \% \mathrm{FC}$ & 41 & 33.2 & 34.12 & 3.93 & 22.60 & 42.85 \\
\hline & $\mathrm{CC} 30 \% \mathrm{FC}$ & 40 & 33.65 & 33.2 & 3.27 & 26.80 & 41.70 \\
\hline & CC20\%FC & 37 & 29.3 & 33.64 & 3.72 & 23.25 & 40.40 \\
\hline & LT & 34.8 & 37.5 & 34.83 & 1.33 & 32.20 & 38 \\
\hline & SDW & 0.130 & 0.16 & 0.16 & 0.049 & 0.039 & 0.272 \\
\hline & Troits & \multicolumn{2}{|c|}{ Means of parents } & \multicolumn{4}{|c|}{ Means of population } \\
\hline & Traits & $\mathbf{S}$ & $\mathrm{V}$ & Mean & SD & Minimum & Maximum \\
\hline \multirow{5}{*}{$\begin{array}{l}\text { Drought stress } \\
\text { condition } \\
(\mathrm{V} \times \mathrm{S})\end{array}$} & RWC & 90.4 & 82 & 73.28 & 10.8 & 52.7 & 92.9 \\
\hline & $\mathrm{CC} 50 \% \mathrm{FC}$ & 41 & 37.1 & 39.04 & 3.29 & 30.7 & 46.2 \\
\hline & $\mathrm{CC} 30 \% \mathrm{FC}$ & 40 & 37.6 & 37.65 & 3.29 & 29.40 & 45.5 \\
\hline & $\mathrm{CC} 20 \% \mathrm{FC}$ & 37 & 35.1 & 38.8 & 4.23 & 25.60 & 47.6 \\
\hline & SDW & 0.130 & 0.122 & 0.14 & 0.039 & 0.065 & 0.24 \\
\hline
\end{tabular}

SD: standard deviation

Table 6. Descriptive statistics for traits in populations of C. Capa $\times S$ and $V \times S$ under normal irrigation (control)

\begin{tabular}{c|c|c|c|c|c|c|c}
\hline & \multirow{2}{*}{ Traits } & \multicolumn{2}{|c|}{ Means of parents } & \multicolumn{4}{c}{ Means of population } \\
\cline { 3 - 8 } & & S & C. Capa & Mean & SD & Minimum & Maximum \\
\hline \multirow{2}{*}{ Normal irrigation } & RWC & 96.7 & 88.37 & 87.93 & 3.25 & 81.38 & 96.7 \\
condition & CC & 37.4 & 34.8 & 34.53 & 4.17 & 22.3 & 44.5 \\
$($ C. Capa $\times$ S) & LT & 34.1 & 34.5 & 32.93 & 1.34 & 29.7 & 37.4 \\
& SDW & 0.322 & 0.262 & 0.27 & 0.085 & 0.098 & 0.475 \\
\hline \multirow{2}{*}{ Traits } & \multicolumn{2}{|c|}{ Means of parents } & \multicolumn{5}{c}{ Means of population } \\
\cline { 3 - 8 } & \multicolumn{2}{|c|}{ S } & V & Mean & SD & Minimum & Maximum \\
\hline Normal irrigation & RWC & 96.7 & 84.7 & 87.61 & 4.8 & 70.74 & 97.21 \\
condition & CC & 37.4 & 35.6 & 36.81 & 3.81 & 25 & 46.15 \\
$(V \times$ S) & SDW & 0.322 & 0.186 & 0.25 & 0.08 & 0.057 & 0.43 \\
\hline
\end{tabular}

SD: standard deviation 


\section{QTL analysis}

Analysis of QTL for physiological traits of C. Capa $\times \mathrm{S}$ has been shown in Table 7 .

Table 7. Identified QTLs in C. Capa $\times$ S population under drought stress in 4-leaf stage

\begin{tabular}{|c|c|c|c|c|c|c|c|}
\hline Trait & $\begin{array}{c}\text { Chromosome } \\
\text { number }\end{array}$ & Position (cM) & LOD & \% Exp. & Left marker & Right marker & $\begin{array}{c}\text { Additive } \\
\text { effect }\end{array}$ \\
\hline RWC & 4 & $37-44$ & 2.88 & 10.7 & E35M55-302\#4-457 & Bmag0384\#4-452 & 3.35 \\
\hline RWC & 5 & $20-53$ & 4.01 & 11.4 & E33M61-110-309 & E33M54-313\#5-374 & 3.52 \\
\hline RWC & 5 & $126-132$ & 3.93 & 14.8 & E41M40-304\#5-411 & E42M51-68-410 & 3.84 \\
\hline CC-50\%FC & 3 & $41-53$ & 4.07 & 13.5 & E33M54-338-152 & E42M55-329\#3-126 & 1.52 \\
\hline CC-50\%FC & 7 & $43-57$ & 3.31 & 11.9 & E37M50-189\#7-194 & E35M55-181\#7-203 & 1.39 \\
\hline $\mathrm{CC}-30 \% \mathrm{FC}$ & 3 & $41-62$ & 3.15 & 14.4 & E33M54-338-152 & E42M55-329\#3-126 & 1.26 \\
\hline $\mathrm{CC}-30 \% \mathrm{FC}$ & 7 & $43-57$ & 2.5 & 10.3 & E37M50-189\#7-194 & E35M55-181\#7-203 & 1.08 \\
\hline CC-20\%FC & 4 & $11-26$ & 3.54 & 9.9 & E39M48-219-446 & E42M50-245-448 & 1.23 \\
\hline CC-20\%FC & 7 & $43-57$ & 2.89 & 8 & E37M50-189\#7-194 & E35M55-181\#7-203 & 1.14 \\
\hline SDW & 1 & $0-30$ & 2.81 & 7.6 & E39M48-310-364 & E38M61-128\#1-363 & 0.020 \\
\hline SDW & 6 & $38-57$ & 3.65 & 12.7 & E33M55-63-76 & E35M61-269\#6-67 & 0.022 \\
\hline SDW & 6 & $145-156$ & 3.61 & 10.5 & E42M48-380\#6-20 & E38M61-197-18 & 0.018 \\
\hline $\mathrm{LT}$ & 4 & $33-44$ & 4.58 & 14.8 & E32M61-166\#4-462 & Bmag0384\#4-452 & 0.547 \\
\hline $\mathrm{LT}$ & 6 & $75-83$ & 2.5 & 9.3 & E33M54-350\#6-41 & E45M49-212-38 & 0.441 \\
\hline $\mathrm{LT}$ & 7 & $0-16$ & 2.95 & 11.9 & E38M55-114\#7-167 & E42M58-388-169 & 0.504 \\
\hline \multicolumn{8}{|c|}{ Identified QTLs in C. Capa $\times \mathrm{S}$ population under normal irrigation (control) } \\
\hline SDW & 3 & $53-62$ & 3.56 & 11.9 & E42M55-329\#3-126 & E39M54-305-113 & -0.036 \\
\hline SDW & 6 & $126-141$ & 3.7 & 13.3 & E37M50-532-24 & E32M55-102-15 & -0.031 \\
\hline
\end{tabular}

There were found a total of 15 QTLs for this population under drought stress. The percentage of phenotypic variance explained by QTLs under drought stress was observed as 7.6, 14.8 and 14.4 for SDW, RWC, LT, respectively. Phenotypic variance ranged from 8 for CC-20\%FC to 14.4 for CC-30\%FC. There were located 15 QTLs for C. Capa $\times \mathrm{S}$ under drought stress on chromosomes 1, 3, 4, 5, 6 and 7. RWC had three QTLs on chromosomes 4 and 5, controlling of only $36.90 \%$ of its phenotypic variance. Also, six QTLs, as two for CC-30\%FC and CC-50\%FC on chromosomes 3 and 7 controlling of $25.4 \%$ and $24.7 \%$, respectively, and two for CC-20\% FC on chromosomes 4 and 7 with $17.9 \%$. SDW covered three QTLs on chromosomes 1 and 6, which controls $31.8 \%$ of its phenotypic variance. There were identified three QTLs on chromosomes 4, 6 and 7 with $36 \%$ of phenotypic variance. For C. Capa $\times \mathrm{S}$ under normal irrigation, two QTLs were found just for SDW on chromosomes 3 and 6 with controlling of 25.2\%, where QTLs were in 53-62 and 126-141 closed to E42M55329\#3-126 and E37M50-532-24 on the left, and E39M54-305-113 and E32M55-102-15 on the right. The highest LOD in C. Capa $\times \mathrm{S}$ was observed in $\mathrm{CC}-30 \% \mathrm{FC}$ as 2.5 , whereas the lowest LOD was recorded in LT as 4.58 in drought stress. In normal irrigating, LOD ranged from 3.56 (for SDW) to 3.7 for (SDW). Increased effect for C. Capa $\times$ S ranged from - 0.018 (for SWD) up to 3.84 (for RWC). Three QTLs on 
chromosomes 1 and 6 for SDW was observed. It has the highest control and impact by closing to E33M55-63-76 and E35M61-269\#6-67. RWC with one QTL and LOD of 4.01 in 20-35 closing to E33M61-110-309 and E33M54-313\#5-374 reached a 3.52increasing effect. 1.52-increasing effect in CC-50\%FC with LOD of 4.07 was recorded for chlorophyll content. Decreasing effect of -0.031 with LOD 3.70 closing to E37M50532-24 and E32M55-102-15 was observed in SDW under normal irrigation. Allelic effect for RWC, CC-50\%FC, CC-30\%FC, CC-20\%FC, SDW, and LT was positive. In contrast, allelic effect was negative for SDW and LT under drought stress and SDW under normal irrigation.

13 QTLs for $\mathrm{V} \times \mathrm{S}$ under a piece of drought stress and normal irrigation were identified (Tables 8 and 9 ).

Table 8. Identified QTLs in $V \times S$ under drought stress in 4-leaf stage of barley

\begin{tabular}{c|c|c|c|c|c|c|c}
\hline Trait & $\begin{array}{c}\text { Chromosome } \\
\text { number }\end{array}$ & $\begin{array}{c}\text { Position } \\
(\mathbf{c M})\end{array}$ & LOD & $\begin{array}{c}\text { \% } \\
\text { Exp. }\end{array}$ & Left marker & Right marker & $\begin{array}{c}\text { Additive } \\
\text { effect }\end{array}$ \\
\hline RWC & 4 & $41-57$ & 4.1 & 12.5 & P17M54-205-301 & E39M61-370-298\#4 & 4.91 \\
RWC & 6 & $106-124$ & 3.34 & 9 & E33M54-129-386 & E38M54-418-203 & 3.60 \\
CC-50\%FC & 3 & $42-54$ & 5.17 & 15.9 & E38M55-322-389\#3 & E33M54-446-351 & 1.32 \\
CC-50\%FC & 3 & $115-136$ & 3.39 & 8.9 & E45M55-449-327 & E39M61-204-323 & 1.06 \\
CC-50\%FC & 7 & $103-132$ & 4.06 & 13.2 & E42M48-283-46\#7 & E42M55-75-52 & 1.31 \\
CC-30\%FC & 1 & $52-79$ & 2.83 & 7.8 & E38M54-313-412 & E33M54-204-82\#1 & 0.99 \\
CC-30\%FC & 2 & $33-38$ & 4.8 & 13.4 & E38M54-241-172\#2 & E35M48-318-186\#2 & 1.34 \\
CC-30\%FC & 5 & $45-63$ & 4.68 & 14.3 & E33M54-313-412 & E42M48-128-424\#5 & 1.29 \\
CC-20\%FC & 2 & $1-28$ & 4.37 & 9.9 & E39M61-184-143 & E38M54-170-154\#2 & 1.72 \\
CC-20\%FC & 3 & $31-50$ & 2.61 & 5.6 & E41M40-106-390 & Bmag0136-382 & 1.11 \\
CC-20\%FC & 3 & $127-136$ & 2.89 & 5.9 & E38M55-264-326\#3 & E39M61-204-323 & 1.19 \\
CC-20\%FC & 6 & $64-82$ & 4.34 & 10 & E38M54-274-221 & E41M40-288-213\#6 & 1.76 \\
SDW & 6 & $85-131$ & 3.23 & 12.8 & E39M48-310-364 & E38M61-128\#1-363 & 0.015 \\
\hline
\end{tabular}

Table 9. Identified QTLs in $V \times S$ under normal irrigation in 4-leaf stage of barley

\begin{tabular}{c|c|c|c|c|c|c|c}
\hline Trait & $\begin{array}{c}\text { Chromosome } \\
\text { number }\end{array}$ & Position (cM) & LOD & \% Exp. & Left marker & Right marker & $\begin{array}{c}\text { Additive } \\
\text { effect }\end{array}$ \\
\hline RWC & 1 & $95-110$ & 3.63 & 10.3 & E35M55-455-120 & E42M55-325-127 & 1.89 \\
RWC & 3 & $37-50$ & 4.72 & 13.9 & E38M55-322-389\#3 & Bmag0136-382 & 2.92 \\
RWC & 3 & $51-96$ & 5.24 & 15.4 & Bmag0136-382 & E39M55-150-332 & 3.35 \\
RWC & 4 & $0-15$ & 3.79 & 9.4 & E38M54-145-199\#4 & E40M32-154-318\#4 & 1.63 \\
CC & 3 & $18-47$ & 3.33 & 5 & E45M55-168-394\#3 & E33M54-129-386 & 1.56 \\
CC & 3 & $50-54$ & 3.24 & 5.6 & E33M54-129 & E32M55-102-15 & 1.62 \\
CC & 4 & $21-35$ & 3.63 & 5.4 & E40M32-200-317 & E41M40-155-302\#4 & 1.51 \\
CC & 4 & $35-50$ & 4.16 & 6 & E41M40-155-302\#4 & E38M55-82-299\#4 & 1.53 \\
CC & 5 & $8-35$ & 4.93 & 7.3 & E45M55-57-406 & P17M54-210-408 & 1.39 \\
CC & 5 & $35-45$ & 4.99 & 7.4 & P17M54-210-408 & E33M54-313-412 & 1.42 \\
CC & 6 & $78-85$ & 3.61 & 5.2 & E33M61-304-214\#6 & E39M61-66-216 & 1.4 \\
SDW & 1 & $0-10$ & 2.71 & 8.8 & E37M50-401-60 & E42M51-232-63 & 0.042 \\
SDW & 7 & $49-60$ & 3.68 & 11.1 & E36M50-316-37 & E38M55-128-39\#7 & 0.032 \\
\hline
\end{tabular}


Phenotypic variance explained by QTLs ranged from 5.6 (for CC-20\%FC) to 15.9 (for CC-50\% FC). Chlorophyll content had the maximum number of QTLs ranging from 5.6 (for $\mathrm{C}-20 \% \mathrm{FC}$ ) to 15.9 (for $\mathrm{CC}-50 \% \mathrm{FC}$ ). There was observed 13 QTLs for $\mathrm{V} \times \mathrm{S}$ under drought stress located on all chromosomes. 10 QTLs were identified for chlorophyll content, three were observed for CC-50\%FC on chromosomes 3 and 7 explaining 38\% of phenotypic variances, and three belongs to chromosomes 1, 2 and 5 with $33.3 \%$, four on chromosomes 2,3 and 6 with $30.4 \%$, and two were determined on chromosomes 4 and 6 with $21.5 \%$. SDW had a QTL on chromosome 6 covering of $12.8 \%$ of its phenotypic variance. Two QTLs were identified on chromosomes 1 and 7 explaining $19.9 \%$ of its phenotypic variance, where they were respectively in $0-10$ and 49-60 closing to E37M50-401-60 and E36M50-316-37 on the left, and E42M51-23263 and E38M55-128-39\#7 on the right. The highest and lowest LOD in V $\times \mathrm{S}$ ranged from 2.61 for $\mathrm{CC}-20 \% \mathrm{FC}$ to 5.17 for $\mathrm{CC}-50 \% \mathrm{FC}$ under drought stress. In contrast, LOD was in a 3.24-5.24 range under normal irrigation. Increasing effect in normal irrigation in $\mathrm{V} \times \mathrm{S}$ ranged from 2.91 for RWC to 3.35 for RWC. The maximum and minimum increasing effect in $\mathrm{V} \times \mathrm{S}$ was recorded as 4.91 and 1.43 for $\mathrm{RWC}$ and $\mathrm{CC}$ $50 \% \mathrm{FC}$, respectively.

One QTL was observed on chromosome 6 for SDW with LOD 3.23 closing to E39M48-310-364 and E38M61-128\#1-363. On the other hand, the highest phenotypic variance for RWC was 15.4\% with LOD 5.24 in the vicinity of Bmag0136-38 and E39M55-150-332 (Table 9). Negative allelic effect was obtained for CC-50\%FC, CC$30 \% \mathrm{FC}, \mathrm{CC}-20 \% \mathrm{FC}$, and SDW under drought stress and also RWC and CC was under normal irrigation.

In C. Capa $\times \mathrm{S}$ under drought stress, there were found great-effect QTLs. For example, a QTL in the vicinity of E33M61-110-309, E33M54-313\#5-374, E41M40304\#5-41, E42M51-68-410 on chromosomes 5 for RWC, a QTL closing to E33M54338-152 and E42M55-329\#3-126 on chromosomes 3 for CC-50\%FC and CC-30\%FC, a QTL in the vicinity of E33M55-63-76 and E35M61-269\#6-67 for SDW, a QTL in the vicinity of E32M61-166\#4-462 and E36M50-155\#4-464 on chromosome 4 for LT. Moreover, the greatest QTL was observed in the vicinity of E37M50-532-24 and E32M55-102-15 on chromosome 6. In $\mathrm{V} \times \mathrm{S}$ under drought stress, a QTL in the vicinity of P17M54-205-301 and E39M61-370-298\#4 on chromosome 4 for RWC, some QTLs closing to E38M55-322-389\#3 and E33M54-446-351 on chromosomes 3 for CC-50\%FC, and a QTL in the vicinity of E38M54-241-172\#2 and E35M48-318$186 \# 2$ on chromosome 2 for CC-30\%FC, and a QTL in the vicinity of E39M48-310364 and E38M61-128\#1-363 on chromosome 6 for SDW. In contrast, in $\mathrm{V} \times \mathrm{S}$ under normal irrigation, major QTLs in the vicinity of Bmag0136-382, E39M55-150-332, E38M55-322-389\#3 and Bmag0136-382 on chromosome 3 for RWC, and a great QTL neighbouring to E36M50-316-37 and E38M55-128-39\#7 on chromosome 7 had the maximum impacts.

Chromosomal map, the number and the type of populations have been presented in Figure 1. Highlighted area on QTLs relates to significant LOD. The areas shown by continuous lines belong to start and end of LOD curve. QTLs in the linkage disequilibrium with same allelic effect had a positive correlation. In contrast, a negative correlation was observed for QTLs in the linkage disequilibrium with different allelic effects. QTLs of CC-50\% FC and $\mathrm{CC}-30 \% \mathrm{FC}$ on chromosome 3, and QTLs CC-30\%FC and CC-20\%FC on chromosome 7 were mapped as a linkage disequilibrium. 

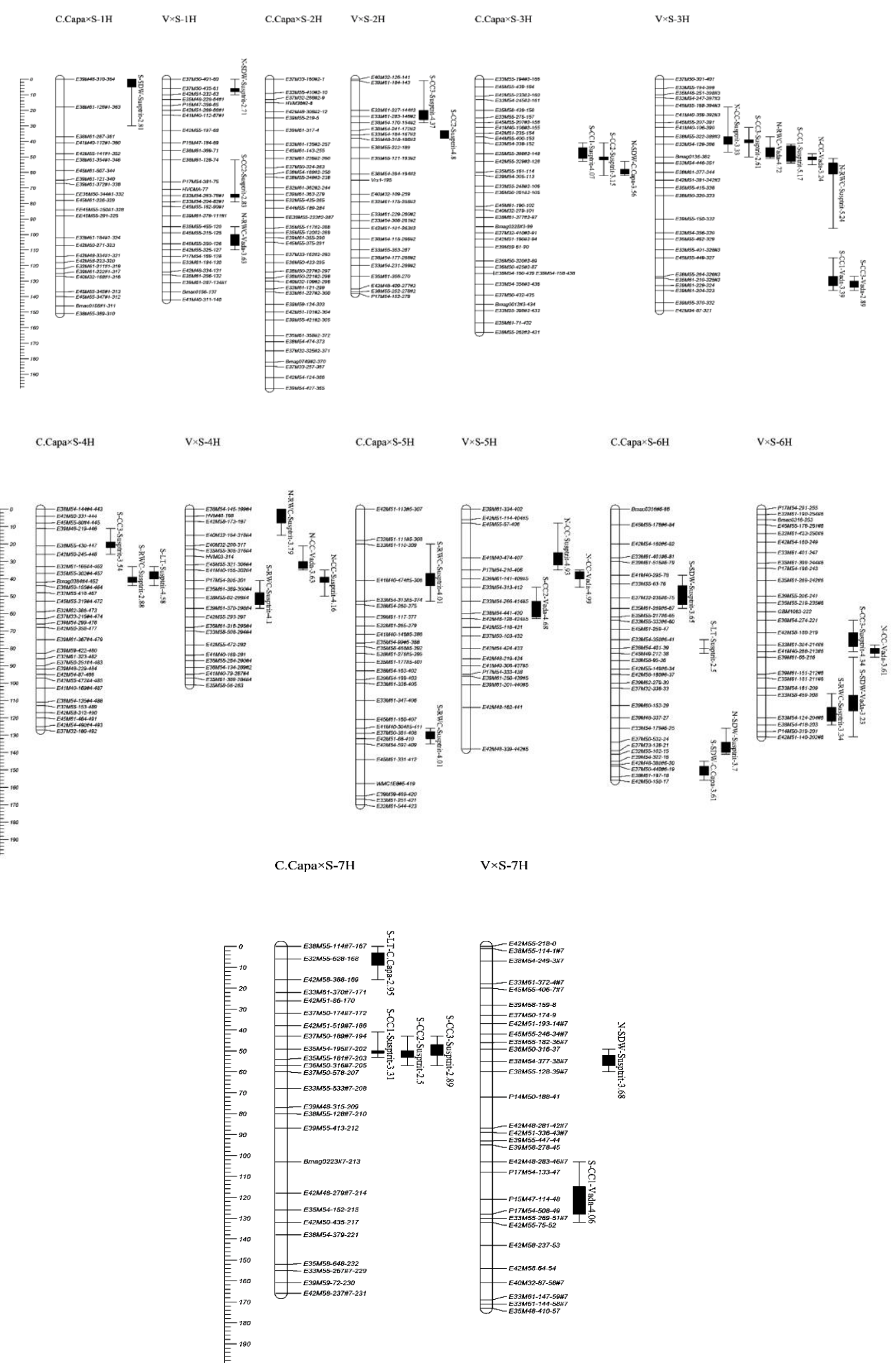

Figure 1. Identified QTLs of physiological traits under drought stress and normal irrigation, and the number and type of the markers. Note: $S$ and $N$ indicate drought stress and normal irrigation, respectively. Susptrit is representative of positive direction of additive effects while

C. Capa and Vada is show negative direction; the numbers relate to maximum LOD.

Highlighted area is corresponded to great LOD. CC1, CC2 and CC3 indicate CC-50\%FC, CC$30 \% F C$ and $C C-20 \% F C$, respectively 


\section{Discussion}

Statistical parameters diversity of traits in both crosses and both environmental conditions (normal and stress) showed that the difference between parents for some traits were significant, shows parents in two extremes. The aggressor separation (positive genetic progress) for the best parent and line was observed. Significant genetic progress in a positive direction and negative indicated that there are increasing and decreasing alleles in parental. For assessing the stability of QTLs, the society should be investigated in different genetic and environmental conditions. In our study, most QTLs mapped to control physiological traits were stable in different conditions and crosses, a finding that agrees with previous studies (Liu et al., 2015; Srividhya et al., 2011). Finding the chromosomal areas controlling the traits is a starting point to use the QTLs in order to improve of the agricultural products. In our study, some genes showed different reactions in various environments based on different sizes and numbers of QTLs. Hence, repeating the experiments in different environments is important, because some QTLs are specialized to given environments. QTL $\times \mathrm{E}$ was indicated as a change in the number of QTLs in different environments or their effects in different environments (Yadav et al., 2003). The QTLs have been reported under drought stress in different studies such as resistance to premature senescence in sorghum (Tuinstra et al., 1997; Crasta et al., 1999), yield in corn (Ribaut and Ragot, 2007), and RWC in barley (Teulat et al., 2001, 2003).

The overlap of traits and identified QTLs on chromosomes may be occurred. In our study, C. Capa $\times \mathrm{S}$ and $\mathrm{V} \times \mathrm{S}$ were conducted in both drought and normal conditions and parent $\mathrm{S}$ was same in both crosses. It was observed for RWC in C. Capa $\times \mathrm{S}$ under drought stress, and in $\mathrm{V} \times \mathrm{S}$ in both drought stress and normal irrigation on chromosome 4. For CC-50\%FC in both $\mathrm{V} \times \mathrm{S}$ and $\mathrm{C}$. Capa $\times \mathrm{S}$ identified QTLs were observed on chromosomes 3 and 7. It was occurred for SDW on chromosome 6. It can be mentioned that according to mutuality of parent $S$ in two crosses, the corresponding QTLs have overlapping areas. QTLs of linkage disequilibrium in the vicinity of each other may reveal a strong correlation between traits. For example, it was observed for RWC, CC$50 \% \mathrm{FC}$, and SDW. The presence of a QTL corresponds to growth in $Q 7 H B$ and $Q 7 H C$ has been reported (Teulat et al., 1997a). The analysis of linkage disequilibrium QTLs can correspond to phenotypic and genotypic correlation. In addition, six QTLs for chlorophyll content in $Q 4 H A, Q 2 H, Q 7 H C, Q 7 H B, Q 5 H C$, and $Q 4 H C$ were mapped in barley (Thisi et al., 2000). This trend was recorded for PHS of barley (Han et al., 2003) and yield of barley (Siahsar and Narouei, 2010). Cluster genes of different traits can make overlap QTLs. Traits controlling the yield should be determined and transfer to high-performance genotypes (Cattivelli et al., 2002). Yang et al. (2007) found a locus under drought stress and five loci under normal irrigation by studying chlorophyll fluorescence of wheat. Baum et al. (2003) identified different QTLs using RAPD and RFLP markers on chromosomes 2, 3, 6 and 7.

Stability of RWC is a significant trait in yield efficiency, which is considered as an eminent factor in tolerance to drought stress (Rezapour Fard et al., 2015). Chen et al. (2010) reported three QTLs for RWC located on chromosomes 1, 2 and 6 in barley under drought stress. 3 QTLs for RWC under early short time drought stress were recorded in barley on chromosomes 2 and 5 (Wójcik-Jagła et al., 2013).

Castro et al. (2008) in QTL analysis of phenological traits for population deriving from $B C D 47$ and Baronesses showed that the parental lines of mapped population have a same growth profile. However, their offspring showed transgressive segregation. In 
the present study, there were found different QTLs whether their location was different or their allelic effect was variable. Drought stress influences physiological traits, which results in creating diversity and instability in QTLs. According to overlapping between QTLs places and mutual parent in both two crosses, we indicated that the QTLs have sufficient stability. The allelic effect in C. Capa $\times \mathrm{S}$ for RWC, CC-50\% FC, CC-30\%FC, CC-20\%FC, SDW and LT was positive under drought stress, whereas it was negative for SDW and LT and SDW under normal irrigation. It shows alleles which decrease SDW and LT, they increase SDW, LT, RWC and CC. A negative allelic effect was observed for $\mathrm{CC}-50 \% \mathrm{FC}, \mathrm{CC}-50 \% \mathrm{FC}, \mathrm{CC}-30 \% \mathrm{FC}, \mathrm{CC}-20 \% \mathrm{FC}$ and SDW under drought stress and for RWC and $\mathrm{CC}$ under normal irrigation. It indicates the alleles which decrease some traits, they can increase others. To understand the controlling areas with more traits is derived from linkage, pleiotropic effect or cluster genes; the high density map is needed (Golshani and Fakheri, 2015). These traits can also easily stabilize through the selection in primary generations of genotypes (Ehdaei and Waines, 1989). QTLs obtained for $C$. Capa $\times S$ and $V \times S$ were stable in most different places. So it can be used in similar findings to select by markers. Due to the controlling of the traits by some loci with high effects, the stability of QTLs is. Stable QTLs relatively stabilize genetic controlling of traits, which cope with QTL $\times$ E.

\section{Conclusions}

Our study showed, there was a significant diversity in two different populations in terms of physiological traits of barley in normal and drought stress conditions. QTLs were distributed on all 7 chromosomes which most of them observed on chromosome 3. Several overlapping regions among QTLs were observed within and between of two populations, that can be related to common parent $S$ in both crosses. QTLs were observed in both positive and negative direction, indicated that there are increasing and decreasing alleles in parents. Stable QTLs were identified, especially for chlorophyll content in different soil moisture conditions. Some QTLs showed different reactions in various environments based on different sizes and numbers. Hence, repeating the experiments in different environments is important, because some QTLs are specialized to unique environments. In both populations, the most QTLs, were related to chlorophyll content, that it seems the trait is more affected from drought stress in barley.

We recommend that the QTLs identified in this study, can be applied in order to marker assisted selection (MAS) and also gene pyramiding, which finally resulted in appropriate genotypes of barley under drought stress. Also chlorophyll content can be used as an important trait in QTL studies under drought stress in barley.

Acknowledgements. We dedicate our special thanks to SZABO LJ. in USDA Agricultural Research Service, Cereal Disease Laboratory, Minneapolis, United States; ALBERTAZZI G. in Universita degli Studi di Modena e Reggio Emilia, Department of Life Science, Modena, Italy; MARCEL TC. in Wageningen University and Research Centre, Wageningen, Netherlands and NIKS, R. E. in Laboratory of Plant Breeding, Wageningen University, Netherlands for their kind cooperation in the preparation of the linkage maps of barley populations and also, we regard to Mehdi Taheri head of Zanjan Agricultural and Natural Resources Research and Education Center, Zanjan, Iran; Karim Mostafavi and other emploees of Tarom Olive Research Station, Tarom, Zanjan, Iran for usfull helps in conduct of this work. 


\section{REFERENCES}

[1] Ashida, T., Nasuda, S., Sato, K., Endo, T. R. (2007): Dissection of barley chromosome 5H in common wheat. - Genes \& Genetic Systems (82): 123-133.

[2] Barr, H. D., Weatherley, P. E. (1962): A re-examination of the relative turgidity technique for estimating water deficit in leaves. - Australian Journal of Biology Sciences (15): 413-428.

[3] Blum, A. (1996): Crop responses to drought and the interpretation of adaptation. - Plant Growth Regulation (20): 135-148.

[4] Castro, A. J., Hayes, P., Viega, L., Vales, L. (2008): Transgressive segregation for phenological traits in barley explained by two major QTL alleles with additivity. - Plant Breeding (127): 561-568.

[5] Cattivelli, L., Baldi, P., Crosatti, C., Fonzo, N. D., Faccioli, P., Grossi, M., Mastrangelo, A. M., Pecchioni, N., Stanca, A. M. (2002): Chromosome regions and stress-related sequences involved in resistance to abiotic stress in Triticeae. - Plant Molecular Biology 48(5-6): 649-665.

[6] Ceccarelli, S. (1987): Yield potential and drought tolerance of segregating populations of barley in contrasting environments. - Euphytica (40): 197-205.

[7] Chen, G., Sagi, M., Weining, S., Krugman, T., Fahima, T., Korol, A. B. (2004): Wild barley eibil mutation identifies a gene essential for leaf water conservation. - Planta 219(4): 684-693.

[8] Chen, G., Krugman, T., Fahima, T., Chen, K., Hu, Y., Röder, M., Nevo, E., Korel, A. (2010): Chromosomal regions controlling seedling drought resistance in Israeli wild barley, Hordeum spontaneum C. Koch. - Genetic Resources and Crop Evolution 57(1): 85-99.

[9] Collard, B. C. Y., Jahufer, M. Z. Z., Brouwer, J. B., Pang, E. C. K. (2005): An introduction to markers, quantitative trait loci (QTL) mapping and marker-assisted selection for crop improvement: the basic concepts. - Euphytica (142): 169-196.

[10] Cooper, M., Eeuwijk, F. A. V., Hamme, G. L., Podlich, D. W., Messina, C. (2009): Modeling QTL for complex traits: detection and context for plant breeding. - Plant Biology (12): 231-240.

[11] Crasta, O. R., Xu, W. W., Rosenow, D. T., Mullet, J., Nguyen, H. T. (1999): Mapping of post-flowering drought-resistance traits in grain sorghum: association between QTLs influencing premature senescence and maturity. - Molecular and General Genetics (262): 579-588.

[12] Ehdaei, B., Waines, J. G. (1989): Genetic variation, heritability and path analysis in land races of bread wheat from South Western Iran. - Euphytica (41): 183-190.

[13] Emberini, L., Michael, P., Moody, D. B., Ogbonnaya, F. C., Black, C. (2009): Pyramiding QTLs to improve malting quality in barley: gains in phenotype and genetic diversity. - Molecular Breeding (23): 219-228.

[14] Golshani, F., Fakheri, B. A. (2015): Mapping genomic regions controlling heavy metals in barley under nickel stress. - Biological Forum - An International Journal 7(1): 12771289.

[15] Gutierrez-Rodriguez, M., Reynolds, M. P., Escalante-Estrada, J. A., Gutierrez-Rodriguez, M. T. (2004): Association between canopy reflectance indices and yield and physiological traits in bread wheat under Drought and well-irrigated conditions. Australian Journal of Agricultural Research (55): 1139-1147.

[16] Hammam Mohammed, K. A. (2004): Improving crop varieties of spring barley for drought and heat tolerance with AB-QTL analysis. - Doctoral Thesis, Institute für Pflanzenbau, Germany.

[17] Han, F., Ullrich, S. E., Romagosa, I., Clancy, J. A., Froseth, J. A., Wesenberg, D. M. (2003): Quantitative genetic analysis of acid detergent fiber content in barley grain. Cereal Science (38): 167-172. 
[18] Jafary, H., Szabo, L. J., Niks, R. E. (2006): Innate non-host immunity in barley to different heterologous rust fungi is controlled by sets of resistance genes with overlapping specificities. - Molecular Plant-Microbe Interactions (19): 1270-1279.

[19] Jafary, H., Albertazzi, G., Marcel, T. C., Niks, R. E. (2008): High diversity of genes for nonhost resistance of barley to heterologous rust fungi. - Genetics (178): 2327-2339.

[20] Liu, L., Sun, G., Ren, X., Li, C. Sun, D. (2015): Identification of QTL underlying physiological and morphological traits of flag leaf in barley. - Genetics 16(29): 1-10.

[21] Mackill, D. J. (2003): What Molecular Tools Are Available for Selection for Drought Tolerance. - In: Fischer, K. S. et al. (eds.). Breeding Rice for Drought-Prone Environments, pp. 55-56. International Rice Research Institute, Los Banos, Philippines.

[22] Nguyen, V. L. (2012): Identification of traits and QTLs contributing to salt tolerance in barley (Hordeum vulgare L.). - Doctoral Thesis, Wageningen University, Netherlands.

[23] Peltonen-Saino, P., Mäkelä, P. (1995): Comparison of physiological methods to assess drought tolerance in oats. - Acta Agriculturae Scandinavica (45): 32-38.

[24] Rezapour Fard, J., Kafi, M., Naderi, R. (2015): The enhancement of drought stress tolerance of Kentucky bluegrass by prohexadione-calcium treatment. - Journal of Ornamental Plants 5(4): 197-204.

[25] Ribaut, J., Ragot, M. (2007): Marker assisted selection to improve drought adaptation in maize: the backcross approach, perspectives, limitations, and alternatives. - Journal of Experimental Botany (58): 351-360.

[26] Siahsar, B. A., Narouei, M. (2010): Mapping QTLs physiological traits associated with salt tolerance in Steptoe $\times$ Morex doubled haploid lines of barley at seedling stage. Journal of Food Agriculture and Environment 8(2): 751-759.

[27] Srividhya, A., Vemireddy, L. R., Venkata Ramanarao, P., Sridhar, S., Jayaprada, M., Anuradha, G., Srilakshmi, B., Reddy, H. K., Hariprasad, A. S., Siddiq, E. A. (2011): Molecular Mapping of QTLs for Drought Related Traits at Seedling Stage under PEG Induced Stress Conditions in Rice. - American Journal of Plant Sciences 2(2): 190-201.

[28] Teulat, B., Monneveux, P., Wery, J., Borries, C., Souyris, I., Charrier, A., Thisi, D. (1997): Relationships between relative water content and growth parameters under water stress in barley: a QTL study. - New Phytologist (137): 99-107.

[29] Teulat, B., Zourmarou-Wallis, N., Rotter, B., Ben Salem, M., Bahri, H., Thisi, D. (2003): QTL for relative water content in field-grown barley and their stability across Mediterranean environments. - Theoretical and Applied Genetics (108): 181-188.

[30] Teulat, B., Borries, C., Thisi, D. (2001): New QTLs identified for plant water status, water-soluble carbohydrate and osmotic adjustment in a barley population grown in a growth chamber under two water regimes. - Theoretical and Applied Genetics (103): 161-170.

[31] Thisi, D., Borries, C., Souyris, I., Teulat, B. (2000): QTL study of chlorophyll content as a genetic parameter of drought tolerance in barley. - Barley Genetics Newsletter (30): 2023.

[32] Tuinstra, M. R., Grote, E. M., Goldsbrough, P. B., Ejeta, G. (1997): Genetic analysis of post-flowering drought tolerance and components of grain development in Sorghum bicolor (L.). - Molecular Breeding 3(6): 439-448.

[33] Wójcik-Jagła, M., Rapacz, M., Tyrka, M., Kościelniak, J., Crissy, K., Żmuda, K. (2013): Comparative QTL analysis of early short-time drought tolerance in Polish fodder and malting spring barleys. - Theoretical and Applied Genetetics 126(12): 3021-3034.

[34] Yadav, R. S., Bidinger, F. R., Hash, C. T., Yadav, Y. P., Yadav, O. P., Bhatnagar, S. K., Howarth, C. J. (2003): Mapping and characterisation QTL $\times$ E interactions for traits determining grain and stover yield millet. - Theoretical and Applied Genetics (106): 512520.

[35] Yang, D. L., Lian, J. R., Ping, C. X., Wei, L. (2007): Quantitative trait loci mapping for chlorophyll Fluorescence and associated traits in wheat (Triticum aestivum). - Journal of Integrative Plant Biology 49(5): 646-654. 